\title{
Novel Optical Fiber Dynamic Light Scattering Measurement System for Nanometer Particle Size
}

\author{
Zhemin Chen, ${ }^{1}$ Pengbing Hu, ${ }^{1}$ Qingqiang Meng, ${ }^{1,2}$ and Xinyong Dong ${ }^{2}$ \\ ${ }^{1}$ Research and Development Centre of Metrology, Zhejiang Province Institute of Metrology, Hangzhou 310013, China \\ ${ }^{2}$ Institute of Optoelectronic Technology, China Jiliang University, Hangzhou 310018, China \\ Correspondence should be addressed to Zhemin Chen; czmasm@hotmail.com
}

Received 19 August 2013; Accepted 19 October 2013

Academic Editor: Sulaiman Wadi Harun

Copyright (c) 2013 Zhemin Chen et al. This is an open access article distributed under the Creative Commons Attribution License, which permits unrestricted use, distribution, and reproduction in any medium, provided the original work is properly cited.

\begin{abstract}
We present a novel optical fiber dynamic light scattering measurement system for nanometer particle size. A multimode fiber probe is used to highly efficiently couple and deliver laser light, while a single-mode fiber probe is used to only receive single scattering light. This design not only improves the measuring ability of the system but also provides flexibility in choosing scattering angle for measurement. By using this system, four kinds of polystyrene nanoparticles are evaluated. Experimentation shows the measured sizes of the nanoparticles are well consistent with the nominal ones, verifying the feasibility of the system.
\end{abstract}

\section{Introduction}

Dynamic light scattering (DLS), called photon correlation spectroscopy (PCS), was first found in the early 1960s [1] and has been widely used in various scientific and engineering fields. It has been discussed theoretically in [2] and was first used for particle size measurement in 1972 [3]. The basic principle of DLS is that the broadening of the intensity line of scattering light can be demodulated for some velocity-related information. Specifically speaking, the translational diffusion coefficient $D$ for the particles in suspension can be derived from the line width of its scattering light. Now, it has become a standard method for submicron particle sizing.

In practical applications, the laser is generally launched on the particles in suspension, and the generating scattering light is detected using standard optical components. In complicated systems, however, local detection of scattering light using DLS technology is sometimes hard to be realized. An effective resolution is to use optical fiber probes to deliver and receive light in DLS system instead of using traditional optical lens. Pioneering work by Brown [4] adopted a couple of single-mode fiber probes, terminated with gradient refractive index lens, to deliver laser light and collect scattering light, respectively. Afterwards, many researchers have attempted to carry out similar experiments of replacing optical lens with fiber probes [5-7]. But in these works, there are few about directly embedding fiber probes in sample solutions, thereby eliminating complexity and inflexibility of goniometers and glass sample holding arrangements in traditional methods.

In recent studies, Chastek presented several miniaturized dynamic light scattering instrumentations comprising two single-mode fiber probes directly embedded into the sample [8]. The construction of the instrumentations permitted the sample volumes to be miniaturized. However, the usage of single-mode fiber reduced the ability of the probes to collect the scattering light, and the scattering angle of the probes was fixed, which to a large extent limited the inflexibility of constructing the instrumentations. Dhadwal used a fiber ferrule design containing single-mode and multimode fiber probes to enhance light collecting capacity [9]. Although this design had the potential to work with high performance and direct contact with the samples, the fabrication of the probe was time consuming and more expensive.

In this work, we proposed and demonstrated a novel optical fiber dynamic light scattering nanometer particle sizing system. Since the fiber probes are used and directly embedded in sample solutions, they provide more flexible measurement for nanoparticles. Moreover, the rational incorporation of multimode fiber elements with single-mode fiber elements in the processing of light improves the spatial coherence 


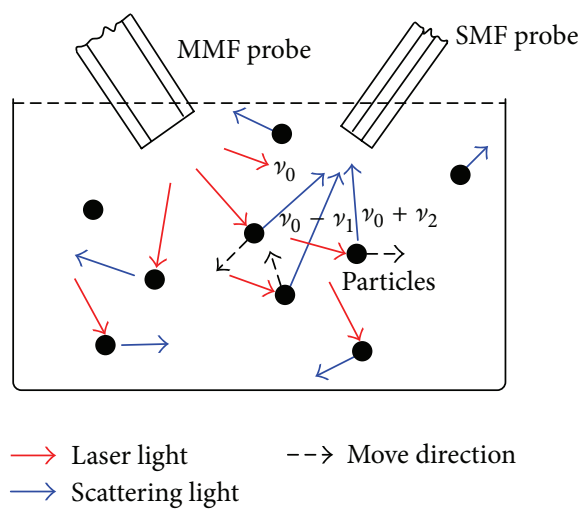

FIGURE 1: Schematic diagram of optical fiber dynamic light scattering measurement.

and thus the measuring ability of the system. Experimental results are in good accordance with the nominal values of the measured nanoparticles, verifying good feasibility of the system.

\section{Operation Principle}

In the proposed DLS system, the information on the nanoparticle size in sample solution is basically acquired by measuring its movement-induced scattering light intensity fluctuations over time. As shown in Figure 1, when laser light delivered by a multimode fiber probe passes through a dilute suspension solution and encounters some moving particles, scattering light will take place. The frequency of the scattering light is relevant to the velocity of the moving particles. Due to random movement of the particles, the frequency and intensity of the scattering light will fluctuate. Therefore, by calculating the fluctuation speed, we can reckon the size of the particles.

Supposing that the movement-induced fluctuations of the scattering light intensity versus time are $I(t)$, the homodyne correlation function, which reflects the dynamics and fluctuations of the scatterers, can be simply expressd as

$$
g_{(1)}(\tau)=\frac{\langle I(t) I(t+\tau)\rangle}{\left\langle I(t)^{2}\right\rangle}
$$

where $\langle\cdot\rangle$ denotes an average over time and $\tau$ is the time delay of the intensity attenuation. Assuming the particles of the dilute suspension solution are monodisperse, that is, all the particles are of the same size and shape, with no interaction between the particles, we can have

$$
\ln \left(g_{(1)}(\tau)\right)=-\Gamma \tau
$$

Here, $\Gamma$ is the decay rate, which is related to the translational diffusion coefficient $D$ of the particles. And they have the following mathematic relationship:

$$
\Gamma=D Q
$$

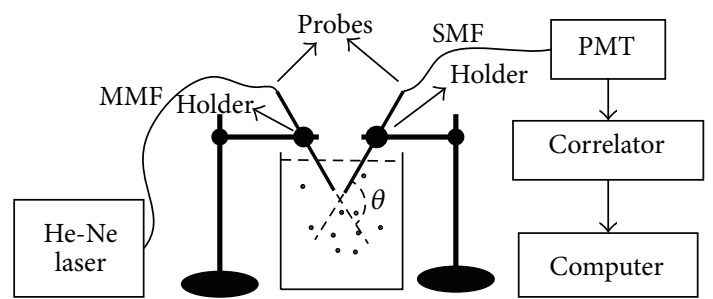

FIgURE 2: Optical fiber dynamic light scattering nanometer particle sizing system.

where the modulus $Q$ of the scattering vector is given by

$$
Q=\frac{4 \pi n_{0}}{\lambda_{0} \sin (\theta / 2)}
$$

Here, $\theta$ is the scattering angle, $\lambda_{0}$ is the laser wavelength in vacuum, and $n_{0}$ is the refractive index of the solvent medium. By analyzing the scattering light intensity, we can calculate $g_{(1)}(\tau)$; thus the average decay rate $\Gamma$ and diffusion coefficient $D$ can be experimentally obtained. With the above estimated values, the particle size of the nanoparticles can be simply determined by Stokes-Einstein relation:

$$
d=\frac{\kappa_{B} T}{3 \pi \eta D} .
$$

Here, $\kappa_{B}$ is Boltzmann constant, $T$ is the absolute temperature of the solution, $\eta$ is the viscosity of the solvent medium, and $d$ is the calculated hydrodynamic diameter of the nanoparticles.

\section{Experimental and Discussions}

The optical fiber DLS system is schematically illustrated in Figure 2. Two fiber probes with stainless steel housing were kept in the sample solution and positioned close to each other. They were mounted inside adjustable probe holders, with a fixed scattering angle $135^{\circ}$ between them. The multimode fiber and fiber probe were selected for more coupling and launching laser light on sampling solution, while the single-mode fiber and fiber probe were selected for avoiding multiple scattering light reception and hence improving the system spatial coherence. The core and cladding diameters of the single-mode fiber are $8 \mu \mathrm{m}$ and $125 \mu \mathrm{m}$, and those of the multimode fibers are $400 \mu \mathrm{m}$ and $440 \mu \mathrm{m}$. This design also provides flexible scattering angle adjustment for nanoparticle sizing.

Light from a $532 \mathrm{~nm}$ He-Ne laser with the power of $100 \mathrm{~mW}$ was coupled into the multimode fiber and was then launched on the measured sample in suspension via the multimode fiber probe. The corresponding scattering light was collected by the single-mode fiber probe and was detected by a photomultiplier tube (PMT, Hamamatsu, H10682-110). The PMT worked in photon counting mode, with the transistortransistor logic (TTL) output connected to a digital correlator (Brookhaven, BI-9000). The final output data were acquired and processed by a computer to demodulate the size of the nanoparticles. 


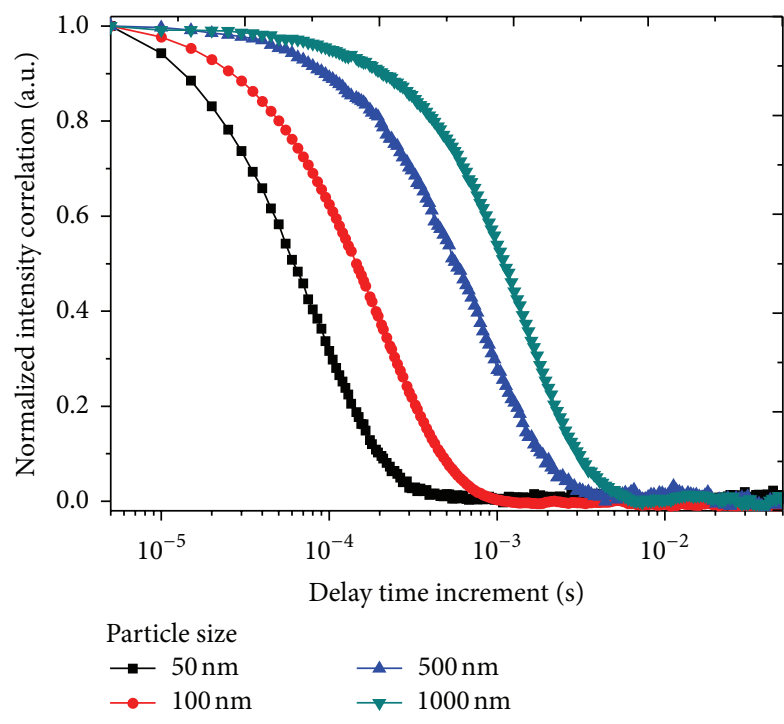

FIGURE 3: Normalized correlation curves of the intensity of scattering light for nanoparticles of different sizes.

The DLS system was set at the minimum sampling speed of $5 \mu \mathrm{s}$ per $2 \mathrm{~min}$. And the measurement was carried out at a constant temperature of $20^{\circ} \mathrm{C}$. Four kinds of polystyrene particles with nominal diameters of $50 \mathrm{~nm}, 100 \mathrm{~nm}, 500 \mathrm{~nm}$, and $1000 \mathrm{~nm}$ (Duke 3000 series) were, respectively, tested by the system.

Figure 3 shows the four sets of the normalized autocorrelation curves of the measured scattering photon intensity from polystyrene particles. From the graph, it can be found that the smaller the nanoparticles are, the faster the autocorrelation curve of the photon intensity declines, which basically agrees with theoretically analyzed ones.

The achieved sizes of polystyrene particles, expressed as the mean values of six repeated measurements, are consistent with the NIST traceable mean diameters of polystyrene particles $(46 \pm 2 \mathrm{~nm}, 102 \pm 3 \mathrm{~nm}, 498 \pm 9 \mathrm{~nm}$, and $1020 \pm 22 \mathrm{~nm})$, as shown in Table 1 . And the corresponding repeatability is $1.17 \%, 2.87 \%, 1.94 \%$, and $1.44 \%$, respectively, verifying good feasibility of the proposed nanoparticle sizing system.

We also studied the influence of the scattering angle on nanoparticle sizing. By adjusting the probe holders, $50 \mathrm{~nm}$ polystyrene particle was able to be measured at four different angles of $165^{\circ}, 150^{\circ}, 135^{\circ}$, and $120^{\circ}$. The results in Table 2 show that there exists good repeatability among the measured particle sizes of different scattering angles; that is to say, we can perform flexible measurement of nanoparticles without precisely adjusting scattering angles. Besides, taking the average value $(47.65 \mathrm{~nm})$ of multiangle measurements as the finally measured size also can effectively eliminate the errors caused by single-angle measurements.

\section{Conclusions}

An optical fiber DLS system for nanoparticle sizing has been presented and demonstrated. A multimode fiber probe and a single-mode fiber probe are both used to improve
TABLE 1: Measured sizes of 4 kinds of polystyrene particles.

\begin{tabular}{ccccc}
\hline Kinds & $46 \pm 2 \mathrm{~nm}$ & $102 \pm 3 \mathrm{~nm}$ & $498 \pm 9 \mathrm{~nm}$ & $1020 \pm 22 \mathrm{~nm}$ \\
\hline Numbers & & & & \\
1 & 46.85 & 103.89 & 491.36 & 998.5 \\
2 & 47.15 & 107.52 & 489.14 & 1009.6 \\
3 & 48.14 & 108.57 & 494.96 & 1003.8 \\
4 & 48.29 & 105.99 & 508.56 & 1017.9 \\
5 & 47.73 & 100.11 & 504.26 & 1023.2 \\
6 & 47.52 & 106.26 & 500.71 & 993.6 \\
\hline Average & 47.613 & 105.39 & 500.165 & 1007.767 \\
\hline
\end{tabular}

TABLE 2: Measured sizes of $50 \mathrm{~nm}$ polystyrene particle versus various scattering angles.

\begin{tabular}{lcccc}
\hline Angles & $165^{\circ}$ & $150^{\circ}$ & $135^{\circ}$ & $120^{\circ}$ \\
Average (nm) & 46.56 & 47.51 & 47.81 & 48.72 \\
Result (nm) & & \multicolumn{2}{c}{47.65} & \\
\hline
\end{tabular}

flexibility and measuring ability of the system. Four kinds of polystyrene nanoparticles are measured by this system, and the results are consistent with the nominal value, verifying good feasibility of the system. The system also has advantages of cost minimization, overall simplicity, and high quality measurements. The study of this system will promote the development and application of optical fiber dynamic light scattering measurement.

\section{Conflict of Interests}

The authors do not have a direct financial relation with the commercial identities mentioned in this paper.

\section{Acknowledgments}

This work was supported by the National Natural Science Foundation of China under Grant no. 61203205 and Special Fund for Quality Inspection Scientific Research in the Public Interest under Grant no. 201210061.

\section{References}

[1] H. Z. Cummins, N. Knable, and Y. Yeh, "Observation of diffusion broadening of rayleigh scattered light," Physical Review Letters, vol. 12, no. 6, pp. 150-153, 1964.

[2] J. Shen, J. C. Thomas, X. Zhu, and Y. Wang, "Wavelet denoising experiments in dynamic light scattering," Optics Express, vol. 19, no. 13, pp. 12284-12290, 2011.

[3] S. P. Lee, W. Tscharnuter, and B. Chu, "Calibration of an optical self-beating spectrometer by polystyrene latex spheres, and confirmation of the Stokes-Einstein formula, Journal of Polymer Science, vol. 10, no. 12, pp. 2453-2459, 1972.

[4] R. G. W. Brown, "Optical fibre sensing using light scattering techniques," Journal of Physics E, vol. 20, no. 11, article 002, pp. 1312-1320, 1987.

[5] S. U. Egelhaaf and P. Schurtenberger, "A fiber-optics-based light scattering instrument for time-resolved simultaneous static and 
dynamic measurements," Review of Scientific Instruments, vol. 67, no. 2, pp. 540-545, 1996.

[6] T. Q. Chastek, K. Iida, E. J. Amis, M. J. Fasolka, and K. L. Beers, "A microfluidic platform for integrated synthesis and dynamic light scattering measurement of block copolymer micelles," Lab on a Chip, vol. 8, no. 6, pp. 950-957, 2008.

[7] D. Pristinski and T. Q. Chastek, "A versatile, low-cost approach to dynamic light scattering," Measurement Science and Technology, vol. 20, no. 4, pp. 1-8, 2009.

[8] T. Q. Chastek, K. L. Beers, and E. J. Amis, "Miniaturized dynamic light scattering instrumentation for use in microfluidic applications," Review of Scientific Instruments, vol. 78, no. 7, Article ID 072201, 8 pages, 2007.

[9] H. S. Dhadwal, "Homodyne fiber optic backscatter dynamic light scattering," Optics Letters, vol. 32, no. 23, pp. 3391-3393, 2007. 

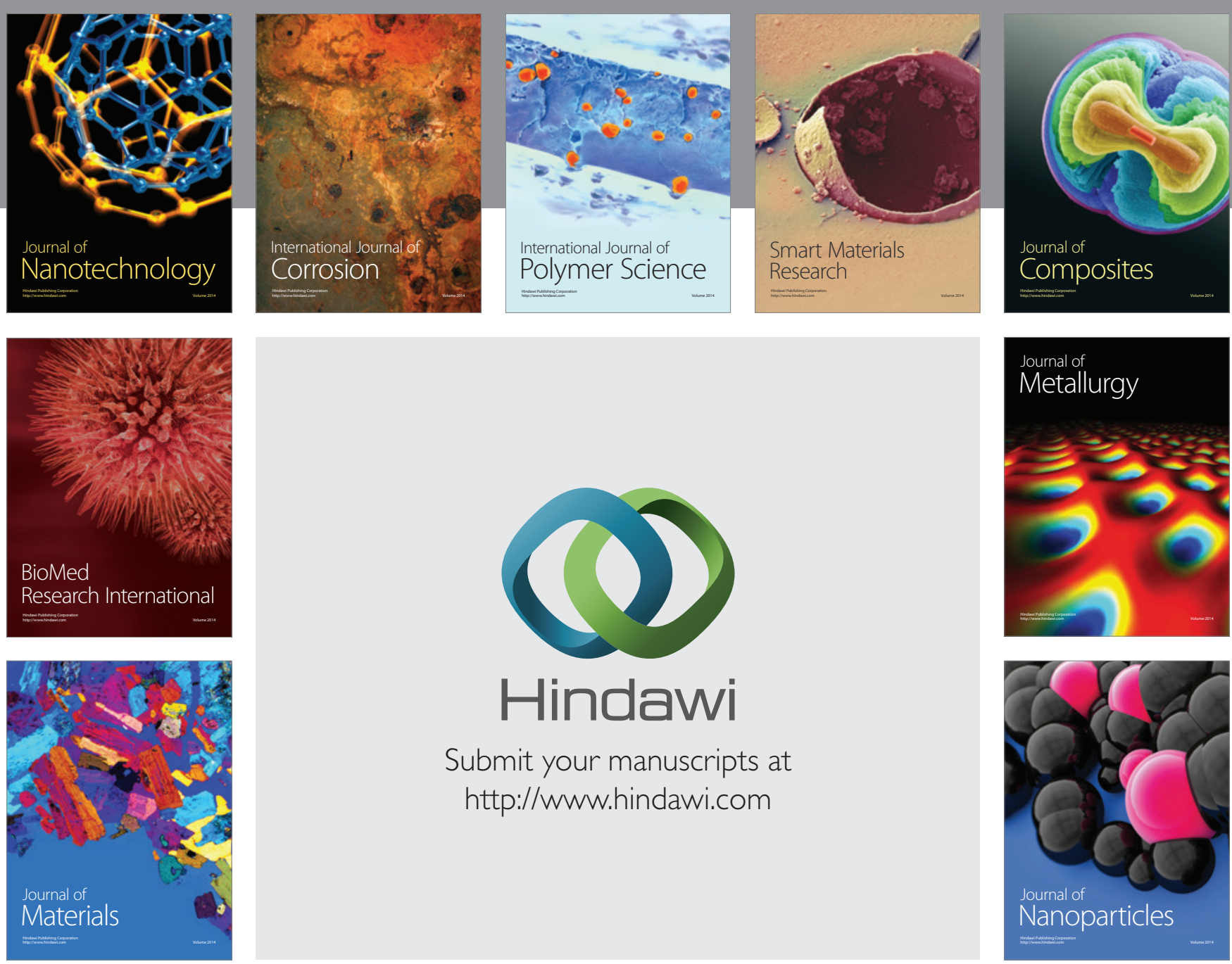

Submit your manuscripts at http://www.hindawi.com
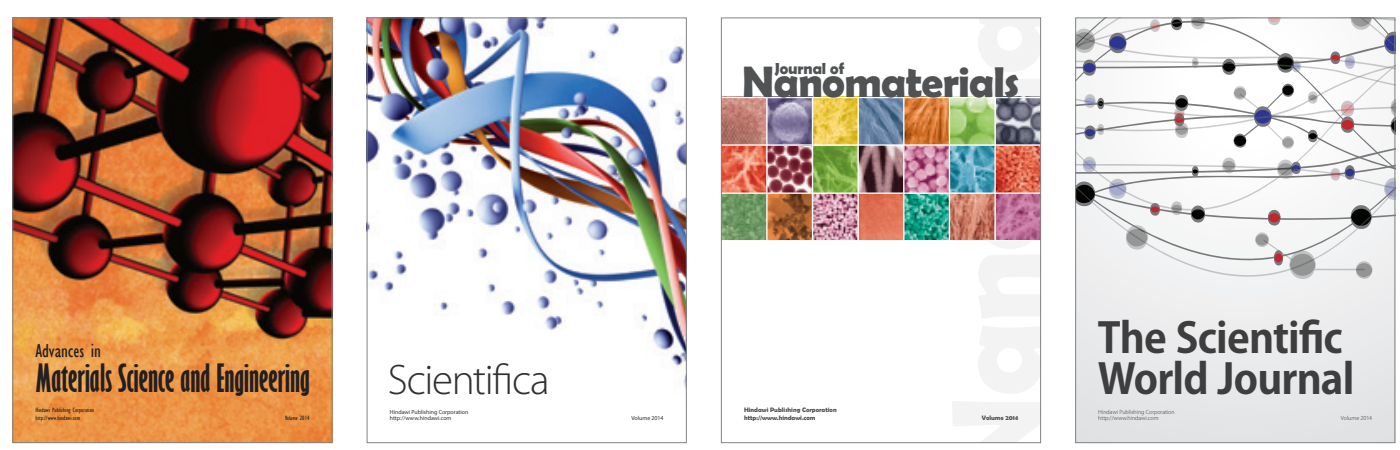

\section{The Scientific World Journal}
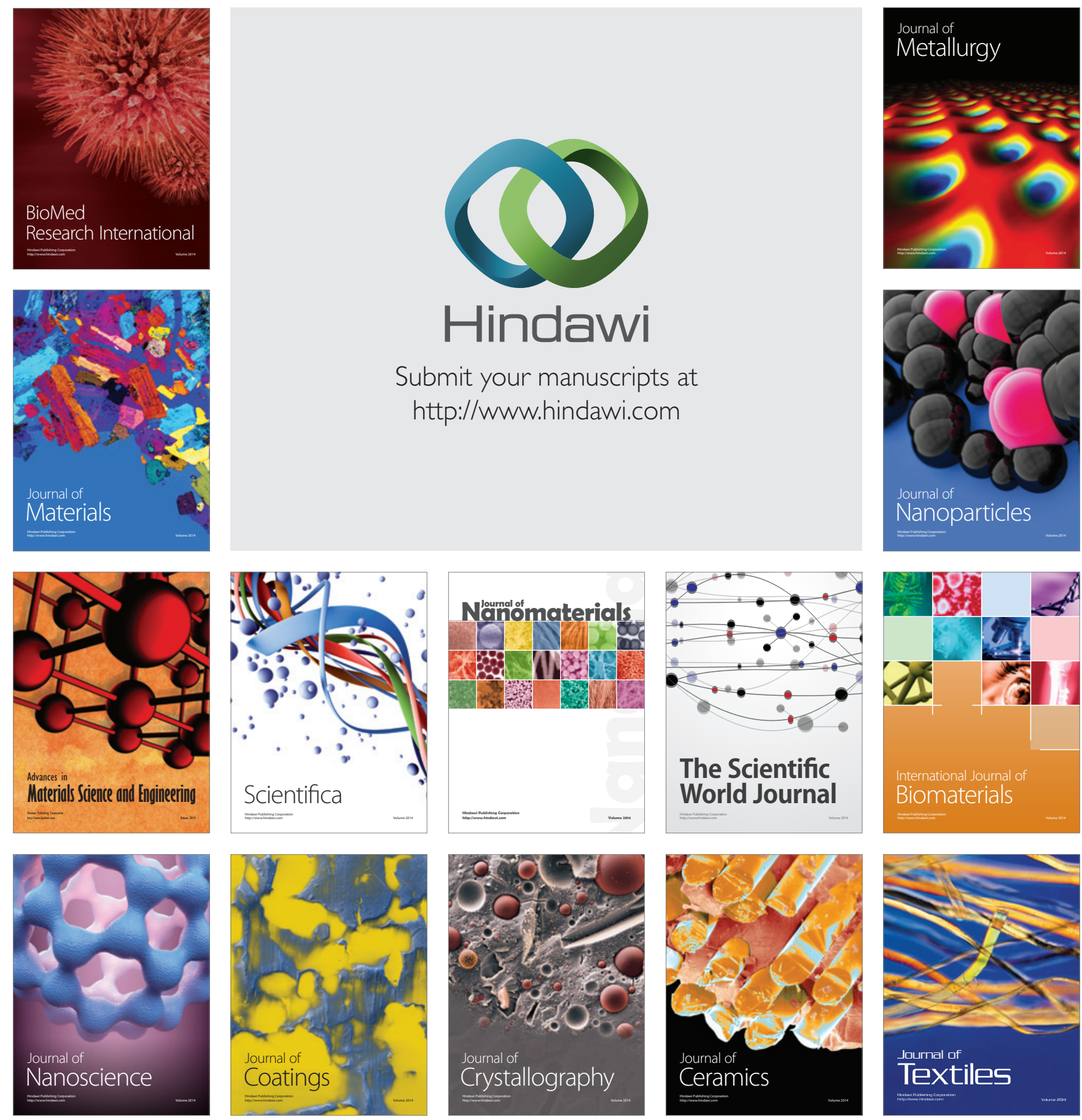